\title{
Autophagic flux blockage in alveolar epithelial cells is essential in silica nanoparticle-induced pulmonary fibrosis
}

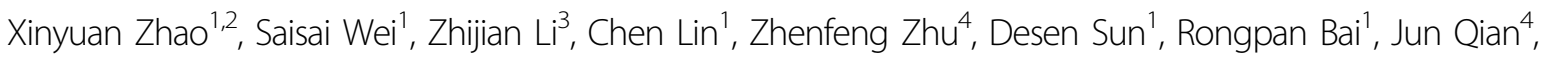
Xiangwei Gao (1)', Guangdi Chen ${ }^{1}$ and Zhengping Xu,

\begin{abstract}
Silica nanoparticles (SiNPs) have been reported to induce pulmonary fibrosis (PF) with an unknown mechanism. Recently, the activation of autophagy, a lysosome-dependent cell degradation pathway, by SiNPs has been identified in alveolar epithelial cells (AECs). However, the underlying mechanism and the relevance of SiNPs-induced autophagy to the development of PF remain elusive. Here, we report that autophagy dysfunction and subsequent apoptosis in AECs are involved in SiNPs-induced PF. SiNPs engulfed by AECs enhance autophagosome accumulation and apoptosis both in vivo and in vitro. Mechanically, SiNPs block autophagy flux through impairing lysosomal degradation via acidification inhibition. Lysosomal reacidification by cyclic-3', $5^{\prime}$-adenosine monophosphate (CAMP) significantly enhances autophagic degradation and attenuate apoptosis. Importantly, enhancement of autophagic degradation by rapamycin protects AECs from apoptosis and attenuates SiNPs-induced PF in the mouse model. Altogether, our data demonstrate a repressive effect of SiNPs on lysosomal acidification, contributing to the decreased autophagic degradation in AECS, thus leading to apoptosis and subsequent PF. These findings may provide an improved understanding of SiNPs-induced PF and molecular targets to antagonize it.
\end{abstract}

\section{Introduction}

Nanoparticles (NPs) defined as particles having at least one dimension below $100 \mathrm{~nm}$ have been applied widely in the last decade in industry and medicine ${ }^{1}$. Among those NPs, silica nanoparticles (SiNPs) are one of the most widely used and closely related to our daily life containing drug delivery, cosmetics and paint, etc ${ }^{2-4}$. The increasing use of NPs has raised concerns about their human and environmental risks. Because their physicochemical properties are different from large particles, NPs may

\footnotetext{
Correspondence: Xiangwei Gao (xiangweigao@zju.edu.cn) or Guangdi Chen (chenguangdi@zju.edu.cn) or Zhengping Xu (zpxu@zju.edu.cn) 'Institute of Environmental Medicine, Zhejiang University School of Medicine, Hangzhou 310058, China

${ }^{2}$ Department of Occupational Medicine and Environmental Toxicology, School of Public Health, Nantong Unversity, Nantong 226019, China

Full list of author information is available at the end of the article.

These authors contributed equally: Xinyuan Zhao, Saisai Wei

Edited by G.M. Fimia
}

potentially result in toxic effects with yet unknown mechamisms. The respiratory system is considered to be one of the main routes by which NPs access human body ${ }^{5}$. Inhalation of these ambient ultrafine particles can result in pulmonary oxidative stress, inflammation, and ultimately cell death ${ }^{1}$. Despite intense investigations, current knowledge of physiological effects of SiNPs on biological barriers and the underlying molecular mechanisms remains fragmented.

Pulmonary fibrosis (PF) is the ultimate result of a large and heterogeneous group of lung disorders known as interstitial lung diseases. It is characterized by excessive accumulation of extracellular matrix, leading to a decline in lung function ${ }^{6}$. Many nano-size materials, including nanoparticulate titanium dioxide, multi- or single-walled carbon nanotubes, as well as SiNPs, have been found to cause $\mathrm{PF}^{7-11}$. The dysregulation of fibroblasts activities including migration, proliferation, secretion, and

\section{(c) The Author(s) 2019}

(c) (i) Open Access This article is licensed under a Creative Commons Attribution 4.0 International License, which permits use, sharing, adaptation, distribution and reproduction cc) in any medium or format, as long as you give appropriate credit to the original author(s) and the source, provide a link to the Creative Commons license, and indicate if changes were made. The images or other third party material in this article are included in the article's Creative Commons license, unless indicated otherwise in a credit line to the material. If material is not included in the article's Creative Commons license and your intended use is not permitted by statutory regulation or exceeds the permitted use, you will need to obtain permission directly from the copyright holder. To view a copy of this license, visit http://creativecommons.org/licenses/by/4.0/. 
myofibroblast differentiation is central to the development of PF. Some NPs, including SiNPs, could activate macrophages to induce inflamatory cytokines secretion ${ }^{7-9}$. These cytokines could triger uncontrolled activation of fibroblasts, which untimately induces PF development. Current paradigms point to alveolar epithelial cells (AECs) injury as another critical event during the pathogenesis of PF. Surrounding the injured AECs, fibroblasts and myofibroblasts form the fibroblastic foci and deposit large amounts of extracellular matrix, thereby destroying the normal alveolar architecture ${ }^{12}$. Although there are studies showing that AECs could uptake NPs in vivo and in vitro, no study has examined the role of AEC damage in NPsinduced $\mathrm{PF}^{13,14}$.

As a genetically programmed pathway for the turnover of cellular components, autophagy has emerged as a crucial process for cellular homeostasis. During autophagy, cytosolic substrate or "cargo" is sequestered into double-membrane vesicle (autophagosome), fusing with lysosome for internal materials degradation ${ }^{15}$. Accumulating evidences suggests that dysregulation of autophagy plays an important role in PF. The mammalian target of the rapamycin (mTOR) signaling pathway, a core signaling pathway to regulate autophagy, has been reported to participate in the process of PF. Using a transgenic mouse model, Gui et al. found that mTOR overactivation in AECs compromised autophagy in the lung and was involved in the pathogenesis of bleomycin-triggered $\mathrm{PF}^{16}$. Similarly, Singh et al. reported that deficient autophagy resulted in upregulation of TGF- $\beta 1$, a key fibrotic driver in PF, promoting PF development ${ }^{17}$. Additionally, autophagy-deficient mice displayed a significantly greater inflammatory response after bleomycin treatment ${ }^{18,19}$. Collectively, these findings support that impaired autophagy may contribute to PF. However, the specific role and underlying mechanism of autophagy, especially in AECs, during NPs-induced PF are still undefined.

In this study, we investigated in detail the dysregulation of autophagy by SiNPs in AECs and defined its contribution to SiNPs-induced PF. Our findings provide the first evidence that SiNPs block autophagic flux in ACEs, contributing to subsequent PF.

\section{Materials and methods}

\section{Synthesis of silica nanoparticles}

The micelles was used to dissolve a certain number of sulfobernteinsaure-bis-2-ethylhexy ester natriumsalz (Aerosol-OT) and 1-butanol in total $10 \mathrm{~mL}$ of DI water under energetic vigorous magnetic stirring. Hundred microliter triethoxyvinylsilane triethoxyvinylsilan (VTES) was added to micellar system mentioned above after $30 \mathrm{~min}$, and was stirred for another $1 \mathrm{~h}$. Then, SiNPs were precipitated after addition of $10 \mu \mathrm{L}$ of (3-aminopropyl) triethoxysilane (APTES) and stirred at room temperature for another $20 \mathrm{~h}$. After successful formation of the SiNPs, excess Aerosol-OT, co-surfactant 1-butanol, VTES, and APTES were removed by dialyzing the solution against DI water in a $12-14 \mathrm{kDa}$ cutoff cellulose membrane for $50 \mathrm{~h}$. The dialyzed solution was then filtered by a $0.45 \mu \mathrm{m}$ filter for further experiments.

\section{Charicterization of silica nanoparticles}

Transmission electron microscope (TEM) was taken by a JEOL JEM-1200EX transmission electron microscope for nanoparticles. $\mathrm{SiO}_{2}$ NPs were dispersed in Roswell Park Memorial Institute (RPMI)-1640 medium (Invitrogen, Carlsbad, CA, USA) for 0 and $24 \mathrm{~h}$ and then subjected to dynamic light scattering (DLS) and zetapotential measurements using the instrument Zetasizer Nano ZS-90 (Malvern Instruments, Orsay, France). Xray diffraction (XRD) was performed using Bruker D8 Advance diffractometer (Bruker, Billerica, MA, USA) and using $\mathrm{CuK} \alpha(\lambda=1.54 \AA)$ as a radiation source.

\section{Plasmids, reagents, and antibodies}

The plasmids GFP-LC3, CFP-LC3, and YFP-LAMP1 were gifts from Dr. Wei Liu (Department of Biochemistry and Molecular Biology, Zhejiang University School of Medicine $)^{20,21}$. The SiNPs were provided by Dr. Jun Qian (State Key Laboratory of Modern Optical Instrumentation, Zhejiang University) and suspended in phosphatebuffered saline (PBS) ${ }^{22}$. Before use, the SiNPs solution was filtered through $0.22 \mu \mathrm{m}$ cutoff membrane filter, then its concentration was detected. To mark SiNPs, Nile Red was added in $\mathrm{it}^{23}$. The chemicals rapamycin (R8781), chloroquine (CQ) (C6628), 3-methyladenine (3-MA) (M9281), cycloheximide (C4859), and EGF (E9644) were from Sigma. TRIzol reagent (3220621) and DNA Transfection Reagent (2102-100) were from SuperFectin. Bodipy-FLpepstatin A (P12271) was from Invitrogen. DND Green189 (L7535) was purchased from Molecular Probes. The blocking serum and Alexa Fluor 488-conjugated goat anti-rabbit second antibody were from Zhongshan Golden Bridge Biotechnology. 8-CPT-cAMP (sc-201569) and IBMX (sc-201188) were from Santa Cruz. The forskolin (S1612) was purchased from Beytime.

The following primary antibodies were used: anti-LC3 (Sigma, L7543), anti-p62 (Sigma, P0067), anti-ACTB (Sigma, A5316), anti-cleaved-Caspase3 (Cell Signaling, 9664), anti-EEA1 (Cell Signaling, 3288), anti-EGFR (Cell Signaling, 4267), anti- $\alpha$-SMA (Proteintech, 14395-1-AP), anti-Bcl-2 (Proteintech, 12789-1-AP), anti-Bax (Proteintech, 23931-1-AP), and anti-CTSD (BD Biosciences, 610800).

\section{Animal treatment}

All animal experiments were performed according to the ethical guidelines by the Ethics Committee of 
Laboratory Animal Care and Welfare, Zhejiang University School of Medicine. To generate pulmonary fibrosis animal model, the Institute of Cancer Research (ICR) male mice at 6 weeks were anesthetized with chloral hydrate, instilled with SiNPs at the dose of $5.0 \mathrm{mg} / \mathrm{kg}$, a dose that has been reported to induce fibrogenic effect ${ }^{24}$, and sacrificed at indicated time. To determine the role of autophagic flux in SiNPs-induced pulmonary fibrosis, mice were given with or without rapamycin $(10 \mathrm{mg} / \mathrm{kg} /$ day after SiNPs instillation). Biopsies of lung tissues were fixed with $10 \%$ paraformaldehyde in sterile phosphatebuffered saline, embedded in paraffin wax, and sectioned at $5 \mu \mathrm{m}$ for further staining. Total protein and RNA from tissues were isolated, and stored at $-80^{\circ} \mathrm{C}$ for determination.

\section{Lung compliance}

Lung compliance was measured by Buxco system (Buxco, RC system). Briefly, mice were anesthetized and placed in a whole body plethysmograph connected to a Harvard rodent ventilator. Data were collected and analyzed using the Biosystems XA software package.

\section{Lung/body coefficient}

Mice were weighted (body weight) before being sacrificed. Their lungs were harvested and weighted (lung wet weight), then the lung/body coefficient was calculated as lung/body coefficient $=$ lung wet weight $[\mathrm{g}] /$ body weight $[\mathrm{g}] \times 100 \%$.

\section{HYP level analysis}

The collagen content in the lung tissue was determined by analysis of the HYP level which was measured by spectrophotometer at $550 \mathrm{~nm}$ with detection kit (Nanjing Jiancheng Bioengineering Institute, A030-2).

\section{Quantitative real-time polymerase chain reaction (qRT- PCR) analysis}

Total RNA was isolated with TRIzol reagent following the manufacturer's protocol. The qRT-PCR analysis was performed as described before ${ }^{25}$. Briefly, real-time quantitative PCR analysis was performed in $10-\mu \mathrm{L}$ reactions using SYBR GREEN PCR Master Mix (Applied Biosystems). The related mRNA level was normalized to the $\beta$ actin mRNA level. The specific primer sequences were listed in Table 1.

\section{Transmission electron microscopy}

After treatment as indicated, lung tissues or A549 cells were fixed in $2.5 \%$ glutaraldehyde at $4{ }^{\circ} \mathrm{C}$ overnight. After dehydration, ultrathin section was embedded and stained with uranyl acetate/lead citrate. Image was captured under a transmission electron microscopy (FEI Company, TECNAI 10).

\begin{tabular}{lll}
$\begin{array}{l}\text { Table } \mathbf{1} \\
\text { reaction }\end{array}$ & DNA sequences of primers for polymerase chain \\
\hline Gene name & Primer name & Sequence \\
\hline Human $\beta-$ & H-ACTB-F & $5^{\prime}$-CACGATGGAGGGGCCGGACTCATC-3' \\
actin & H-ACTB-R & $5^{\prime}$-TAAAGACCTCTATGCCAACACAGT-3' \\
Human $L C 3 b$ & H-LC3b-F & $5^{\prime}$-TTGGTGAACGGACACAGCAT-3' \\
& H-LC3b-R & $5^{\prime}$-CGTCTCCTGGGAGGCATAGA-3' \\
Mouse $\beta$-actin & M-ACTB-F & $5^{\prime}$-GCAGATGTGGATCAGCAAGC-3' \\
& M-ACTB-R & $5^{\prime}$-AGCTCAGTAACAGTCCGCC-3' \\
Mouse CTGFF & M-CTGF-F & $5^{\prime}$-AGACCTGTGCCTGCCATTAC-3' \\
& M-CTGF-R & $5^{\prime}$-ACGCCATGTCTCCGTACATC-3' \\
\hline
\end{tabular}

\section{Masson, Sirius red, TUNEL assay, and}

immunohistochemistry staining

The tissue slice with $5 \mu \mathrm{m}$ thickness was subjected to Masson staining or Sirius red staining for identification of collagen fibers ${ }^{26}$. Apoptosis cells in the tissue section were detected by TUNEL using IHC assay with a commercial apoptosis kit (11684817910, Roche), according to the supplier's instruction. For IHC staining, the tissue section was deparaffinized and rehydrated, and boiled in $10 \mathrm{mM}$ citrate buffer for $30 \mathrm{~min}$ for antigen retrieval. Endogenous peroxidase was blocked with 3\% hydrogen dioxide for $10 \mathrm{~min}$. The slide was blocked in $3 \%$ bovine serum albumin (BSA) at room temperature for $1 \mathrm{~h}$, incubated with corresponding primary antibody (LC3, 1:1000; p62, 1:200; or $\alpha$-SMA, $1: 200)$ at $4{ }^{\circ} \mathrm{C}$ overnight, then incubated with anti-rabbit secondary antibody for $1 \mathrm{~h}$, and visualized with 3,3-diaminobenzidine substrate (Zhongshan Golden Bridge Biotechnology, \#PV-9000-D). Finally, the section was counterstained with hematoxylin.

\section{Cell culture, transfection, and autophagy induction}

The human lung epithelial cells (A549) were kindly provided by Dr. Yuehai Ke (Department of Basic Medical Sciences, Zhejiang University School of Medicine), and were grown as a monolayer in Royal Park Memorial Institute (RPMI) 1640 (Invitrogen, C22400500BT) supplemented with $10 \%$ fetal bovine serum (FBS, Gibco, $1027-106)$ at $37^{\circ} \mathrm{C}$ in an humidified atmosphere of $95 \%$ air and $5 \% \mathrm{CO}_{2}$. Transient transfection was performed using DNA Transfection Reagent (Invitrogen, 11668-019) according to manufacturer's instruction.

For autophagy induction, the cells were incubated in starvation medium (1\% BSA, $140 \mathrm{mM} \mathrm{NaCl}, 1 \mathrm{mM} \mathrm{CaCl}_{2}$, $1 \mathrm{mM} \mathrm{MgCl}, 5 \mathrm{mM}$ glucose, and $20 \mathrm{mM}$ HEPES, $\mathrm{pH} 7.4$ ) at $37^{\circ} \mathrm{C}$ for $2 \mathrm{~h}$, or cultured in $10 \%$ FBS of RPMI 1640 with $200 \mathrm{nM}$ rapamycin for $24 \mathrm{~h}$. 


\section{Immunoblotting analysis}

Total proteins were extracted from cells or lung tissue after lysed with lysis buffer, quantified by bicinchonininc acid (BCA) protein assay kit (Beyotime, P0009), and applied to immunoblotting analysis as described previously ${ }^{27}$. The equal amounts of total proteins were separated by sodium dodecyl sulfate polyacrylamide gel electrophoresis and transferred to nitrocellulose membrane. The membrane was blocked with 3\% BSA in TBST buffer for $2 \mathrm{~h}$. Next, the blot was incubated with corresponding primary antibody (anti-LC3, 1:1000; anti-p62, 1:1000; anti-EGFR, 1:1000; antiACTB, 1:2000; anti-GAPDH, 1:2000; or anti-CTSD, 1:500) at $4{ }^{\circ} \mathrm{C}$ overnight followed by incubation with IR-Dye conjugated secondary antibody (Goat anti-Mouse, Li-COR Biosciences, B80908-02, 1:10,000; or Goat anti-Rabbit, LiCOR Biosciences, B81009-02, 1:10,000) for $1 \mathrm{~h}$. Finally, the specific bands were analyzed using an Odyssey infrared imaging system (Li-COR Biosciences) and then quantified using densitometry.

\section{Immunofluorescence staining}

The cells cultured on coverslips were washed three time in $1 \times$ PBS, fixed with $4 \%$ paraformaldehyde for $15 \mathrm{~min}$ at $4{ }^{\circ} \mathrm{C}$, and permeabilized with $0.2 \%$ Triton X-100 for 15 min at $4{ }^{\circ} \mathrm{C}$. The non-specific binding was blocked by goat serum (Zhongshan Golden Bridge Biotechnology, \#ZLI9021) at room temperature for $2 \mathrm{~h}$. Then the cells were incubated with primary antibodies at $4{ }^{\circ} \mathrm{C}$ overnight (antiLC3, 1:200; anti-p62, 1:200; anti-EEA1, 1:100; anti-EGFR, 1:1000), and incubated with Alexa Fluor 488-conjugated goat anti-rabbit second antibody (1:1000) for $1 \mathrm{~h}$. The sample was further incubated with 4',6-diamidino-2phenylindole (DAPI, Sigma, D9542) for $15 \mathrm{~min}$ to stain nuclei. Finally, the cover slip was mounted onto a glass slide with $87 \%$ glycerol, and the immunofluorescence staining was visualized with a confocal microscope (Nikon, A1R).

\section{EGFR degradation assay}

The cells treated with SiNPs at concentration of $50 \mu \mathrm{g} /$ $\mathrm{mL}$ for $24 \mathrm{~h}$ were incubated in serum-free DMEM with $10 \mu \mathrm{M}$ cycloheximide for another $4 \mathrm{~h}$. Then, the cells were treated with $100 \mathrm{ng} / \mathrm{mL}$ EGF for different durations. The EGFR expression level was evaluated by cellular immunofluorescence assay, or immunoblotting assay.

\section{Determination of lysosomal acidification}

For lysosomal acidification assay, the cells were incubated with the $1.0 \mu \mathrm{M}$ DND Green-189 (Invitrogen, L7525) for $30 \mathrm{~min}$ at $37^{\circ} \mathrm{C}$. After staining, cells were washed with PBS twice, and cellular fluorescence was evaluated by flow cytometry (Beckman Coulter, FC500MCL) or imaged by a fluorescent microscope (Nikon, ECLIPSE Ti) to quantify lysosomal acidification.

\section{Bodipy-FL-pepstatin A staining}

For mature CTSD staining, the cells were washed with PBS for twice, and were incubated with $1 \mathrm{mM}$ Bodipy-FLpepstatin A for $1 \mathrm{~h}$ at $37^{\circ} \mathrm{C}$. After washing with PBS, cells were imaged by a confocal microscope (OLYMPUS, BX61W1-FV1000) to quantify relative pepstatin A intensity.

\section{Lysosomal reacidification}

To reacidify lysosome, a cAMP cocktail was used. It consisted of 8-CPT-cAMP $(500 \mu \mathrm{M})$, IBMX $(100 \mu \mathrm{M})$, and forskolin $(10 \mu \mathrm{M})$.

\section{Cell viability analysis}

The effects of various treatments on cell viability were determined by Cell Counting Kit-8 (CCK-8) reagent (DOJINDO, CK04). Briefly, $10 \mu \mathrm{L}$ of CCK8 reagent was added to each well of cells plated in 96-well plate and incubated for another $2 \mathrm{~h}$ at $37^{\circ} \mathrm{C}$. The optical density (OD) at $450 \mathrm{~nm}$ was measured by using a Microplate Reader (Thermo Scientific, VaripsKan Flash).

\section{Apoptosis assay}

Apoptosis analysis was performed by Annexin V staining. Briefly, cells were resuspended and incubated with Annexin V (MultiSciences Biotech Co., Ltd, 5300117) for $5 \mathrm{~min}$ in the dark. The sample was analyzed by flow cytometry (Beckman Coulter, FC500MCL), and the percentage of Annexin $\mathrm{V}$ positive cells was counted.

\section{Statistical analysis}

The SiNPs size was measured by Image J software. The fluorescence intensities and protein colocalization coefficients were quantified by the MetaMorph software. The 3D structure and size measurement of lysosome were carried out with Imaris software. Comparison between two groups (Control and SiNPs) for statistical difference were performed with Student's two-tailed $t$ test. One-way ANOVA was used to determine statical significance for multiple groups analysis. For lysosome volume, Wilcoxon rank sum test was used. All statistical analyses were performed using GraphPad Prim version 6.0. A $P$ value $\leq 0.05$ was considered significant. Differences were considered statistically significant if $P<0.05{ }^{(*)}, P<0.01\left(^{(* *)}\right.$, or not significant (NS).

\section{Results}

\section{SiNPs induce PF in mice}

We prepared silica SiNPs as described in methods with an average diameter about $26.85 \mathrm{~nm}$ (Fig. 1a). The DLS of SiNPs in culture media RPMI-1640 were $37.53 \pm 1.98 \mathrm{~nm}$, and exhibited good stability in media since the size did not increase after $24 \mathrm{~h}$ of incubation $(38.12 \pm 2.37 \mathrm{~nm}$, Table 2). In addition, the results of zeta potentials of 
A

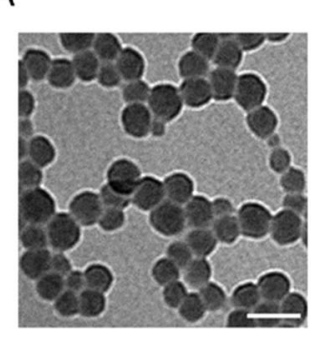

C

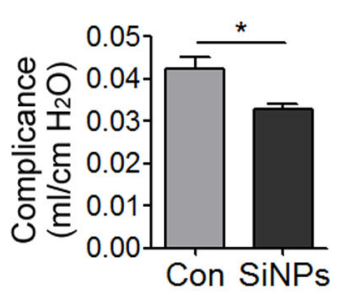

F

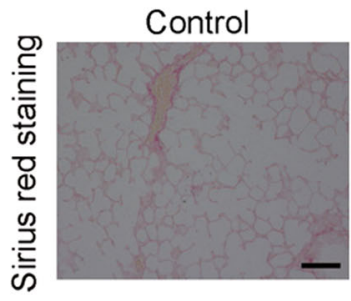

I

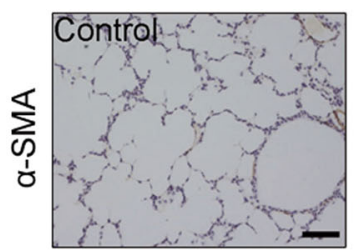

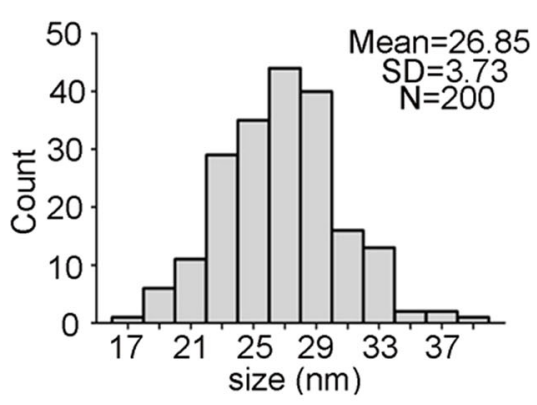

D

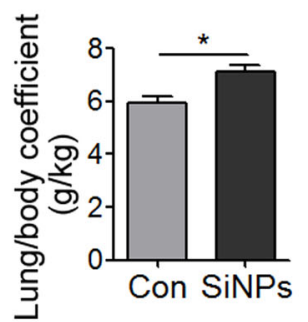

E
B

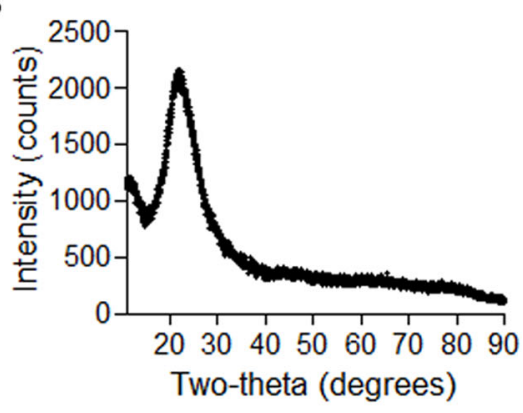

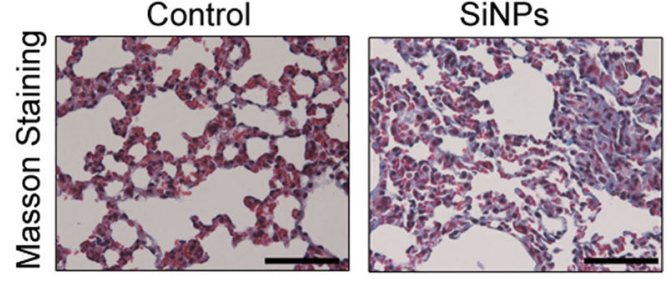

G

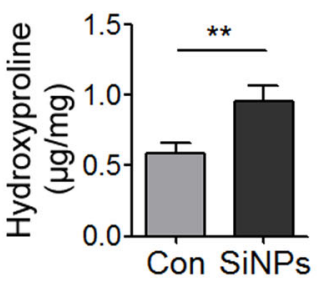

$\mathrm{H}$
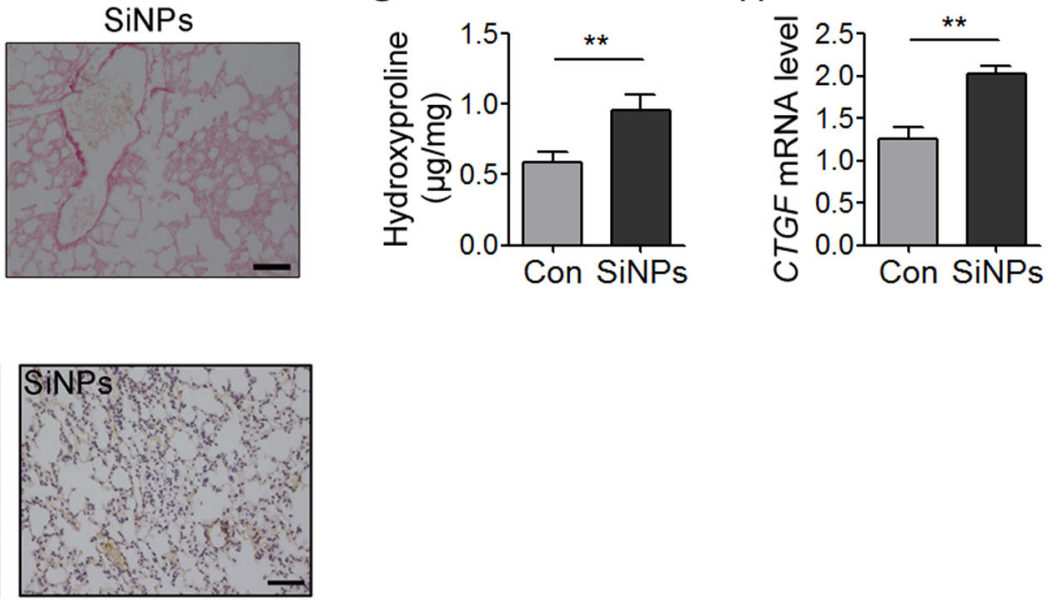

Fig. 1 SiNPs induce pulmonary fibrosis (PF). a SiNPs were imaged by TEM. SiNPs size showed approximately normal distribution, and the average SiNPs size was about $26.85 \mathrm{~nm}$. Scale bar: $50 \mathrm{~nm}$. b XRD analysis of SiNPs using CuKa $(\lambda=1.54 \AA)$ as a radiation source. $\mathbf{c}-\mathbf{i}$ The mice were instilled once with SiNPs and sacrificed 1 month later. $\mathbf{c}$ Lung compliance was determined by Buxco system. $\mathbf{d}$ The weights of lung and body were recorded to determine the lung/body coefficient. $\mathbf{e}, \mathbf{f}$ The lung tissue sections were stained by Masson staining (e) or Sirius red staining (f) to identify collagen fibers. $\mathbf{g}$ The hydroxyproline (HYP) content in lung tissue was assessed by spectrophotometry. $\mathbf{h}$ The relative CTGF mRNA level was measured by qRTPCR. $\mathbf{i}$ The expression level of a-SMA was detected by immunohistochemistry (IHC). Data are presented as mean \pm SEM $(n=5$ for each group). * $P<$ $0.05,{ }^{* *} P<0.01$. Scale bars: $100 \mu \mathrm{m}$

SiNPs in our study were $-30.40 \pm 0.83$ and $-28.75 \pm$ $0.59 \mathrm{mV}$, respectively. The X-ray diffraction pattern of the silica nanoparticles indicates a peak at $2 \theta=22^{\circ}$, which reveals the amorphous nature of the silica nanoparticles. Further, the XRD pattern confirms the absence of any ordered crystalline structure (Fig. 1b).

To determine whether these SiNPs could induce PF, mice were instilled with SiNPs at $5 \mathrm{mg} / \mathrm{kg}$. The mice were sacrificed at one month after instillation, and lung compliance and lung/body coefficient, as important indexes of lung function, were evaluated. The results showed that comparing to the control group, pulmonary compliance was significantly reduced (Fig. 1c), and lung/body coefficient was significantly increased in SiNPs-treated group (Fig. 1d), indicating an impairment of lung function. Furthermore, Masson's staining and Sirius red staining revealed an increased collagen deposition in SiNPsexposed mice lung tissue (Fig. 1e, f). SiNPs treatment significantly increased the expression of hydroxyproline (HYP), a major constituent of collagen, and connective 
tissue growth factor (CTGF), which could induce collagen synthesis, in mice lungs (Fig. 1g, h), further demonstrating collagen accumulation in lung tissues ${ }^{28}$. Finally, $\alpha$-smooth muscle actin ( $\alpha$-SMA) expression level, as active fibrotic indicator, was significantly upregulated in the lung tissues of SiNPs-treated mice (Fig. 1i) ${ }^{29}$. Taken together, these data indicate that SiNPs do induce PF in mice under current experimental conditions.

Table 2 DLS, zeta potential, and polydispersity index of SiNPs in RPMI 1640 at different time points

\begin{tabular}{llll} 
Time (h) & DLS (nm) & Zeta potential $(\mathbf{m V})$ & Polydispersity index \\
\hline 0 & $37.53 \pm 1.98$ & $-30.40 \pm 0.83$ & $0.295 \pm 0.03$ \\
24 & $38.12 \pm 2.37$ & $-28.75 \pm 0.59$ & $0.301 \pm 0.04$ \\
\hline
\end{tabular}

\section{SiNPs enter AECs via endosome/lysosome pathway}

To test the hypothesis that the AECs damage contributes to SiNPs-induced PF, we first examined that AECs can endocytose SiNPs. The transmission electron microscopy (TEM) image showed that SiNPs were in type II AECs one day after instillation (Fig. 2a). To further confirm our observation, A549 cells were exposed to SiNPs at a concentration of $50 \mu \mathrm{g} / \mathrm{mL}$ for $24 \mathrm{~h}$ and imaged by TEM. As expected, A549 cells also engulfed SiNPs (Fig. 2b). Next, we monitored the subcellular localization of SiNPs at different time points. The results showed that SiNPs mainly colocalized with early endosome antigen 1 (EEA1), an early endosome marker, after 30 min treatment (Figure S1A, Supporting Information). With the treatment time extends, more and more SiNPs were colocalized with lysosomal-associated membrane protein 1 (LAMP1), a lysosome marker, accompanied by

A
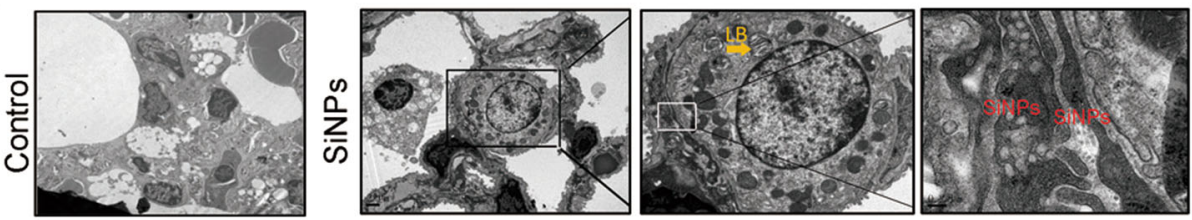

B
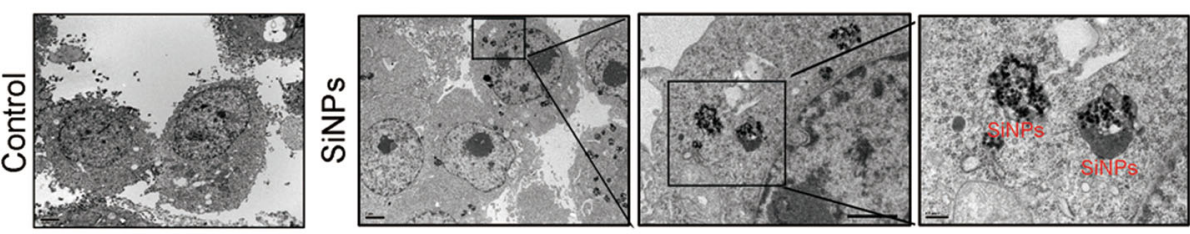

C
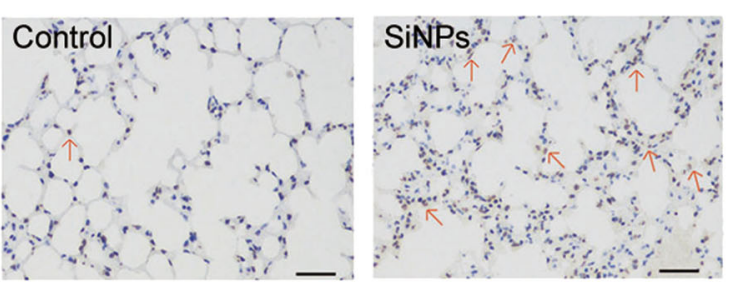

D
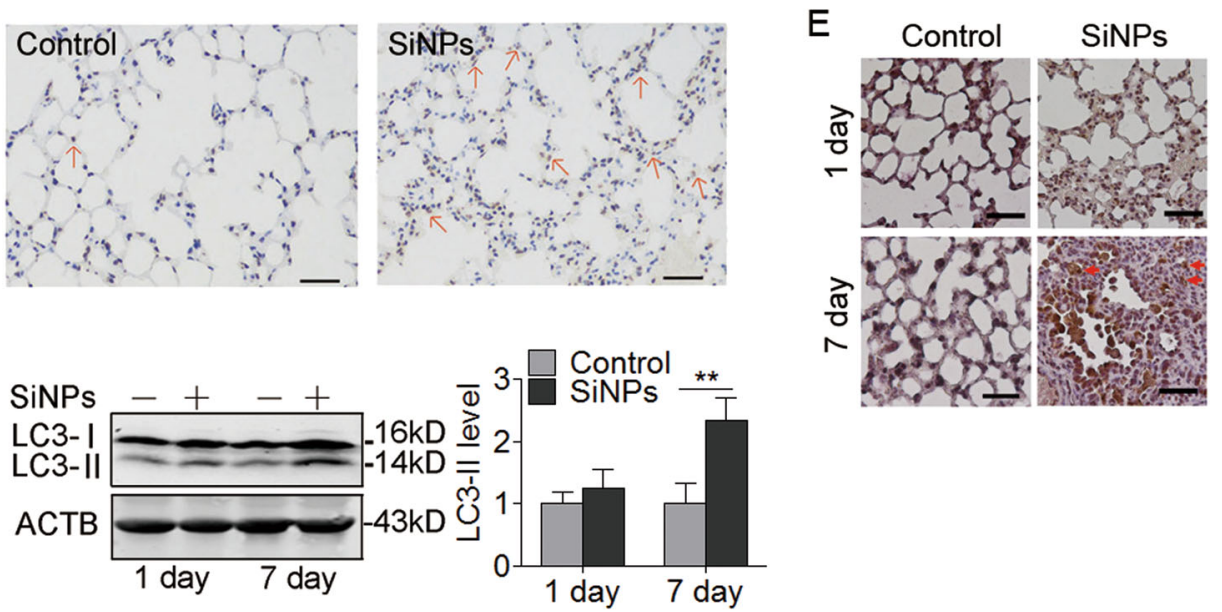

Fig. 2 SiNPs induce apoptosis and autophagosome accumulation in mouse lung tissue. a The mice were instilled once with SiNPs (100 $\mu$ g) and harvested 1 day later. The lung tissue was imaged by TEM. LB, lamellar body. Scale bars: $200 \mathrm{~nm}$. b The cellular location of SiNPs in A549 was imaged by TEM. Scale bars: $200 \mathrm{~nm}$. c Cell apoptosis for lung tissue from mice with or without SiNPs treatment was detected by TUNEL assay 1 month after instillation. Red arrows: TUNEL-positive cells. Scale bars: $100 \mu \mathrm{m}$. d, e The mice were instilled with PBS or SiNPs, and harvested at the indicated time. d LC3 protein expression of lung tissue was detected by western blot, and the right panel shows the statistics for the relative LC3-II protein level. e LC3 expression was detected by IHC at the indicated time after instillation. Scale bars: $100 \mu m$. All the quantitative data are presented as mean \pm $\operatorname{SEM}\left(n=5\right.$ for each group), ${ }^{* *} P<0.01$ 
decreased colocalization with EEA1 (Figure S1B, Supporting Information), suggesting a translocation from early endosome to lysosome. These data indicated that SiNPs could penetrate AEC membrane and enter the lysosomes via endosome/lysosome pathway.

\section{SiNPs stimulate autophagosome accumulation and cell apoptosis both in vitro and in vivo}

To reveal the biological consequence of cellular SiNPs, cell apoptosis was determined by terminal deoxynucleotidyl transferase-mediated nick end labeling (TUNEL) assay in vivo. The result showed obvious TUNEL-positive staining in SiNPs-treated group, indicating that SiNPs exposure induced cell apoptosis in lung tissue (Fig. 2c). Next, we detected the expression level of microtubule-associated protein 1 light chain 3 (MAP1LC3), an autophagosome marker, in lung tissues by Western blot, and found that LC3-II expression was increased in SiNPs-treated mice (Fig. 2d). Immunohistochemistry (IHC) staining confirmed that the LC3 protein accumulated in lung tissues from SiNPs-treated mice (Fig. 2e).

We further performed in vitro studies using A549 cells. The results showed that SiNPs increased LC3-II expression in a dose-dependent manner in A549 cells (Fig. 3a). SiNPs-induced LC3-II expression was also observed in another lung epithelial cells BEAS-2B (Figure S2A). Consistently, immunofluorescence (IF) staining revealed more LC3-positive punctuated structures in SiNPstreated cells (Fig. 3b). Similarly, increased green fluorescent protein (GFP) tagged LC3 (GFP-LC3) punctuated structures were found in GFP-LC3-expressing A549 cells after SiNPs treatment (Fig. 3c). SiNPs induced significant autophagosome accumulation within the first $3 \mathrm{~h}$ trearment (Fig. 3d). Higher concentration of SiNPs $(50 \mu \mathrm{g} / \mathrm{ml})$ also upregulated the espression of B-cell lymphoma 2 (Bcl-2)-associated X protein (Bax), an indicator of apoptosis (Fig. 3e). In addition, exposure to SiNPs led to apoptosis in a dose-dependent way as indicated by gradually increased Bax (Fig. 3f). Taken together, these data demonstrate that SiNPs induce autophagosome accumulation and apoptosis in AECs.

\section{SiNPs inhibit autophagosome degradation}

To further confirm that SiNPs trigger autophagy, the protein sequestosome 1 (p62), a substrate that is preferentially degraded by autophagy, was detected. Unexpectedly, the results revealed that SiNPs led to increase rather than decrease of $\mathrm{p} 62$ protein level in a dose- and time-dependent way (Fig. 4a, S2B), and that p62 proteins were assembled into aggregates in SiNPs-treated cells (Fig. 4b), suggesting that autophagic degradation is inhibited upon SiNPs treatment. Therefore, we performed an autophagic flux assay in the presence or absence of a lysosome degradation inhibitor, chloroquine (CQ). The results showed that $C Q$ treatment did not further increase the LC3-II levels in SiNPs-treated cells (Fig. 4c, S2C), implying the blockage of autophagic flux by SiNPs.

We next directly evaluated the degradation of autophagosomes by applying 3-methyladenine (3-MA) to inhibit the synthesis of new autophagosomes, and detected LC3-II protein levels. Under 3-MA treatment, LC3-II was dramatically degraded in starved cells. However, LC3II degradation was inhibited in SiNPs-treated cells (Fig. 4d). Autophagosome degradation was also tracked by time-lapse confocal microscopy in GFP-LC3 expressing cells. Similarly, we found that GFP-LC3 puncta were retained in SiNPs-treated cells after 3-MA treatment, further confirming an inhibition of autophagosome degradation (Fig. 4e). Thus, our data demonstrate that SiNPs block autophagic flux and autophagic degradation.

\section{SiNPs impair lysosomal degradation through inhibiting lysosomal acidification}

After autophagosome formation, the complete autophagic process comprises their fusion with lysosomes and subsequent cargo degradation. We, therefore, first evaluated autophagosome-lysosome fusion by observing the colocalization of cyan fluorescent protein (CFP) tagged LC3 (CFP-LC3) and yellow fluorescent protein (YFP) tagged LAMP1. The results showed that the colocalization of LC3 and LAMP1 in SiNPs-treated cells was almost equal to starved cells (Fig. 5a), suggesting that SiNPs do not affect autophagosome-lysosome fusion. We then evaluated lysosomal degradation using an epidermal growth factor receptor (EGFR) degradation assay. The result revealed that EGFR puncta were largely retained in SiNPs-treated cells while a significant portion of the internalized EGFR was degraded in the control group (Fig. 5b, c). The immunoblotting assay also showed that SiNPs retarded cellular EGFR degradation (Fig. 5d), confirming a dysfunction of lysosomal degradation. These data indicate that SiNPs impair lysosomal degradation capacity.

We further investigated the mechanism involved in lysosome impairment. The results revealed that the lysosome size was significantly increased (Fig. 6a, b), indicating a lysosome swelling. We next examined lysosomal acidification, a critical factor for lysosome maturation and activation of most lysosomal enzymes. Fluorescent GreenDND 189, a pH-dependent lysosensor, was introduced to the cells, and the fluorescence intensity was evaluated by flow cytometry. SiNPs treatment decreased the fluorescence intensity of Green-DND 189 (Fig. 6c, S2D), suggesting an increased lysosome $\mathrm{pH}$. We further investigated the maturation of lysosomal enzymes under inhibition of acidification. Lysosomal hydrolase cathepsin D (CTSD) is a representative lysosomal enzyme, and 
A

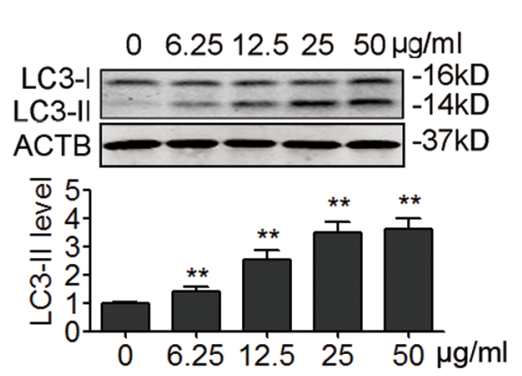

C

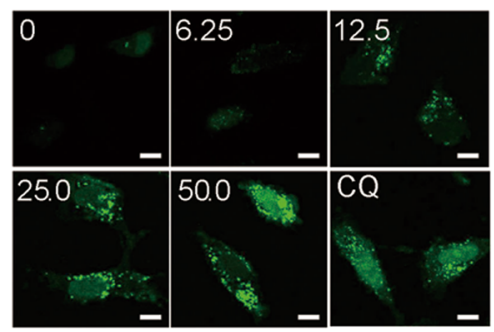

D

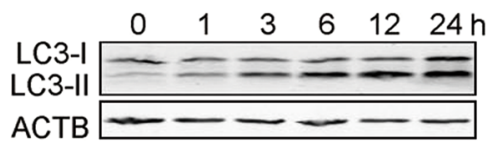

E
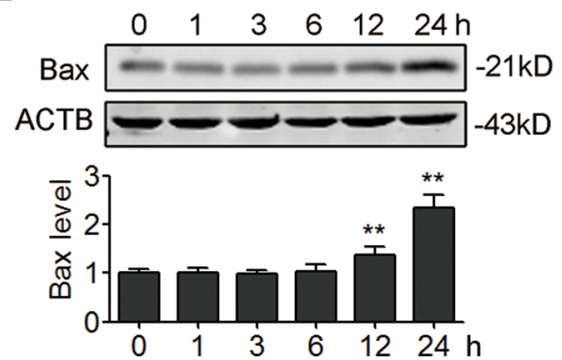

B
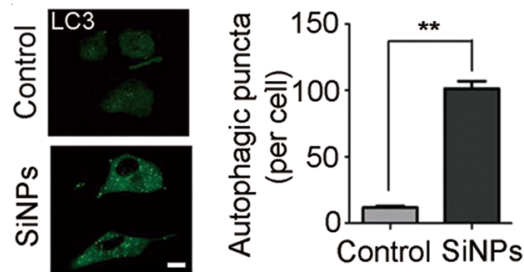
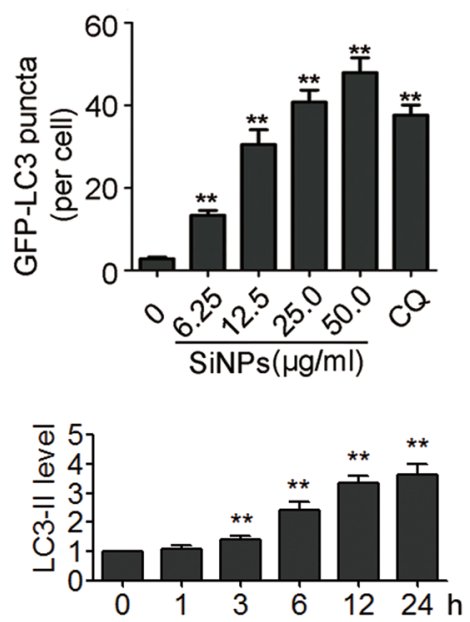

$\mathrm{F}$
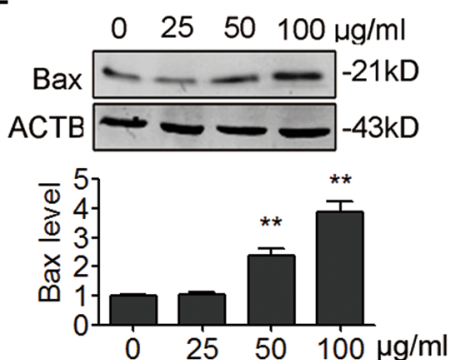

Fig. 3 SiNPs stimulate autophagosome accumulation and apoptosis in A549 cells. a A549 cells were treated with different concentrations of SiNPs for $24 \mathrm{~h}$, and the cellular LC3 expression levels were detected by western blot. The bottom panel shows the statistics for the relative LC3-II protein level. b A549 cells were treated with SiNPs at $50 \mu \mathrm{g} / \mathrm{mL}$ for $24 \mathrm{~h}$, and the cellular LC3 expression level was evaluated by indirect immunofluorescence (IF) assay. Quantification shown on the right represents the average number of autophagic puncta per cell. $n=50$. $\mathbf{c}$ A549 cells transiently expressing GFP-LC3 were exposed to different concentrations of SiNPs for $24 \mathrm{~h}$, or treated with $20 \mu \mathrm{M}$ chloroquine (CQ) for $6 \mathrm{~h}$ as a positive control, and were imaged by confocal microscopy. The graph on the right shows the statistics for the average number of GFP-LC3 puncta per cell. $n=50$. $\mathbf{d}$ A549 cells were treated with SiNPs at $50 \mu \mathrm{g} / \mathrm{mL}$ for different time as indicated, and cellular LC3 expression was assessed by western blot. The right panel shows the statistics for the relative LC3-II protein level. e A549 cells were treated with SiNPs at $50 \mu \mathrm{g} / \mathrm{mL}$ for different time as indicated, and cellular Bax expression was assessed by western blot. The bottom panel shows the statistics for the relative Bax protein level. $\mathbf{f}$ A549 cells were treated with different concentration of SiNPs for $24 \mathrm{~h}$, and cellular Bax expression was assessed by western blot. The bottom panel shows the statistics for the relative Bax protein level. ${ }^{* *} P<0.01$. Scale bars: $5 \mu \mathrm{m}$

Bodipy-FL-pepstatin A, a fluorescence dye, could selectively bind to mature active $\mathrm{CTSD}^{30}$. We found that mature CTSD levels were significantly decreased in SiNPs-treated cells (Fig. 6d). Immunoblotting analysis also demonstrated that SiNPs treatment resulted in a decline of mature CTSD protein level (Fig. 6e). These data indicate that SiNPs impair lysosomal acidification.

\section{Increasing autophagic degradation protects cells from SiNPs-triggered apoptosis}

To test whether the inhibition of autophagic degradation accounts for SiNPs-induced apoptosis, we performed rescue experiment. We used intracellular signaling molecule cyclic- $3^{\prime}, 5^{\prime}$-adenosine monophosphate (cAMP) to restore lysosomal $\mathrm{pH}$, and then evaluated lysosomal 
A
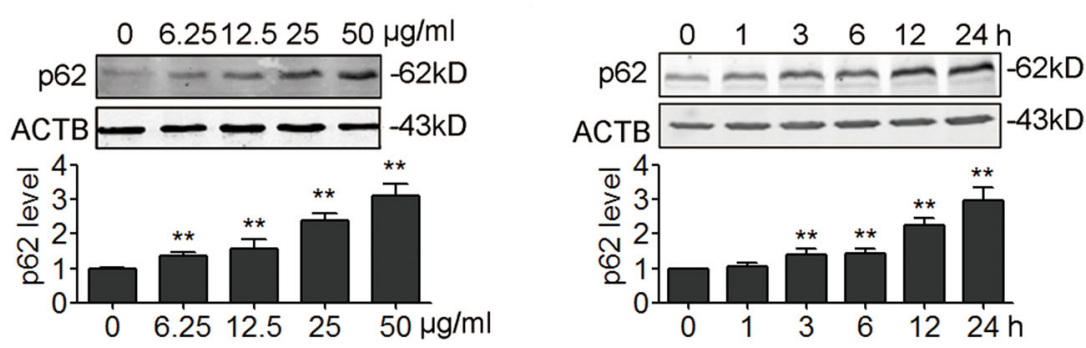

B

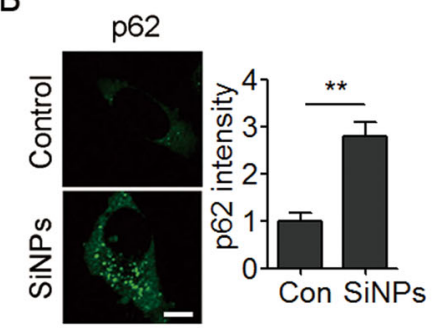

$\mathrm{C}_{\mathrm{SiNPS}}-+\overline{+}+$
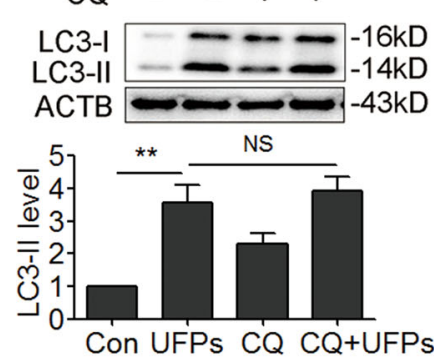

D

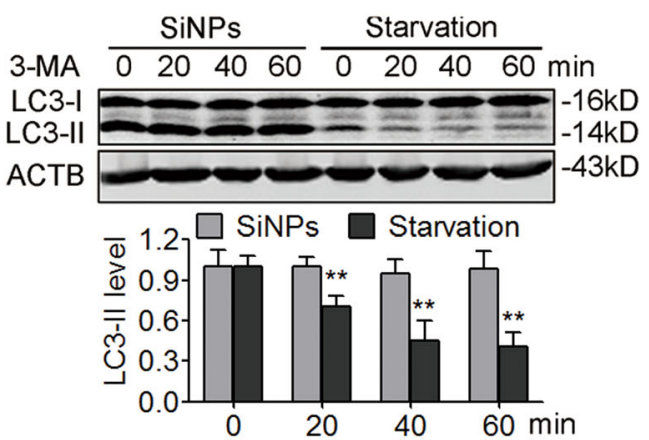

$\mathrm{E}$

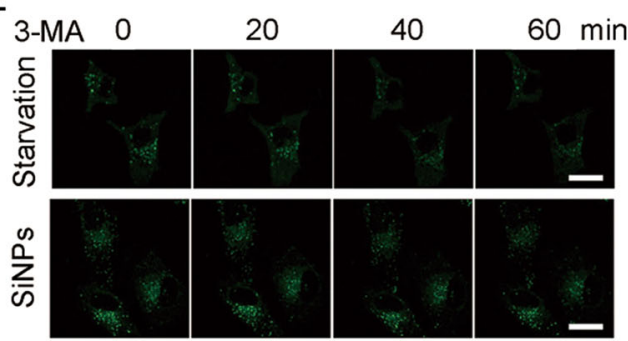

Fig. 4 SiNPs inhibit autophagic degradation. a A549 cells were treated with different concentration of SiNPs as indicated for $24 \mathrm{~h}$ or with $50 \mathrm{\mu g} / \mathrm{mL}$ of SiNPs for different time as indicated. The cellular p62 expression was detected by western blot. The bottom panel shows the statistics for the relative p62 protein level. b A549 cells were treated with SiNPs at $50 \mu \mathrm{g} / \mathrm{mL}$ for $24 \mathrm{~h}$. The expression of p62 was evaluated by indirect immunofluorescence assay. Quantification shown on the right represents the relative fluorescence intensity of p62. Scale bar: $5 \mu \mathrm{m}, n=50$. c A549 cells were treated with SiNPs at $50 \mu \mathrm{g} / \mathrm{mL}$ in the absence or presence of CQ $(20 \mu \mathrm{M})$ for $24 \mathrm{~h}$, and the LC3 protein level was detected by western blot. The bottom panel shows the statistics for the relative LC3-II protein level. d A549 cells were treated with SiNPs at $50 \mu \mathrm{g} / \mathrm{mL}$ for $24 \mathrm{~h}$ or starvation medium, and then treated with $10 \mathrm{mM}$ of 3-MA. The LC3 protein level was assessed by western blot at the indicated time. The bottom panel shows the statistics for the relative LC3-II protein level. e A549 cells transiently expressing GFP-LC3 were treated with 3-MA as in (d), and imaged by confocal microscopy. Scale bars: $10 \mu \mathrm{m}$. All quantitative data are presented as mean $\pm \mathrm{SEM}$, ${ }^{* *} P<0.01$

acidification, autophagic flux, and apoptosis ${ }^{31}$. Addition of cAMP indeed reduced lysosomal $\mathrm{pH}$, and enhanced maturation of CTSD in SiNPs-treated cells (Figure S3A,B, Supporting Information). Furthermore, p62 and LC3-II levels in SiNPs-treated cells were significantly reduced in the presence of cAMP (Fig. 7a), suggesting that the reacidification of lysosomes enhances autophagic flux and promotes autophagic degradation. After cAMP treatment, cell viability loss (Figure S3C, Supporting Information), as well as cell apoptosis in SiNPs-treated cells were significantly attenuated (Fig. 7b).

Finally, we tested if a decreased autophagic degradation could directly account for apoptosis by enhancement of degradation with rapamycin. The results showed that rapamycin reduced p62 protein levels in A549 cells and significantly inhibited SiNPs-induced apoptosis (Fig. 7c, d). In addition, rapamycin also attenuated SiNPs-induced apoptosis in vivo (Fig. 7e, f). Collectively, our data indicate that SiNPs-induced apoptosis in AECs is a result of impared autophagic degradation.

\section{Decreased autophagic degradation participates in SiNPs- induced PF}

The observation that inhibition of autophagic degradation in A549 cells contributed to apoptosis implies a new mechanism underlying the pathogenesis 


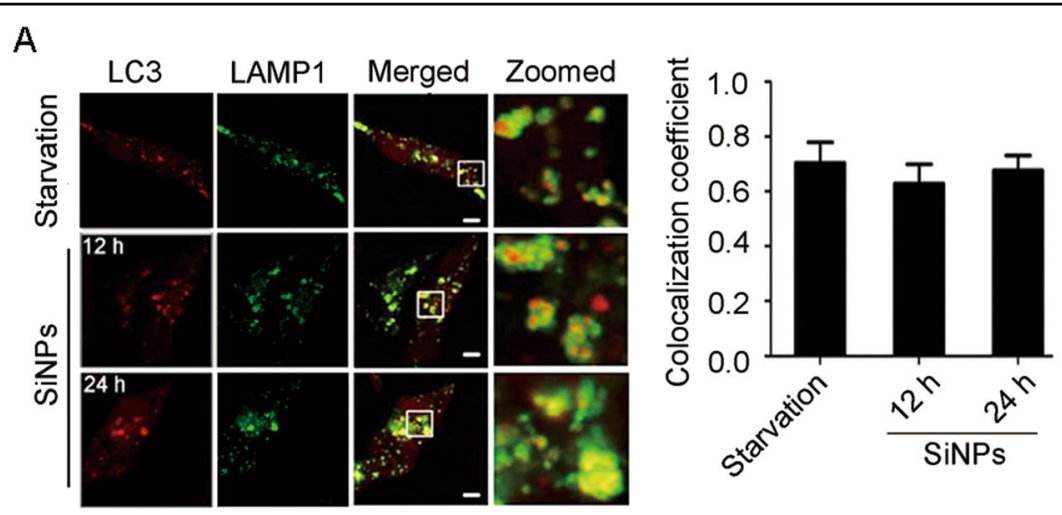

B

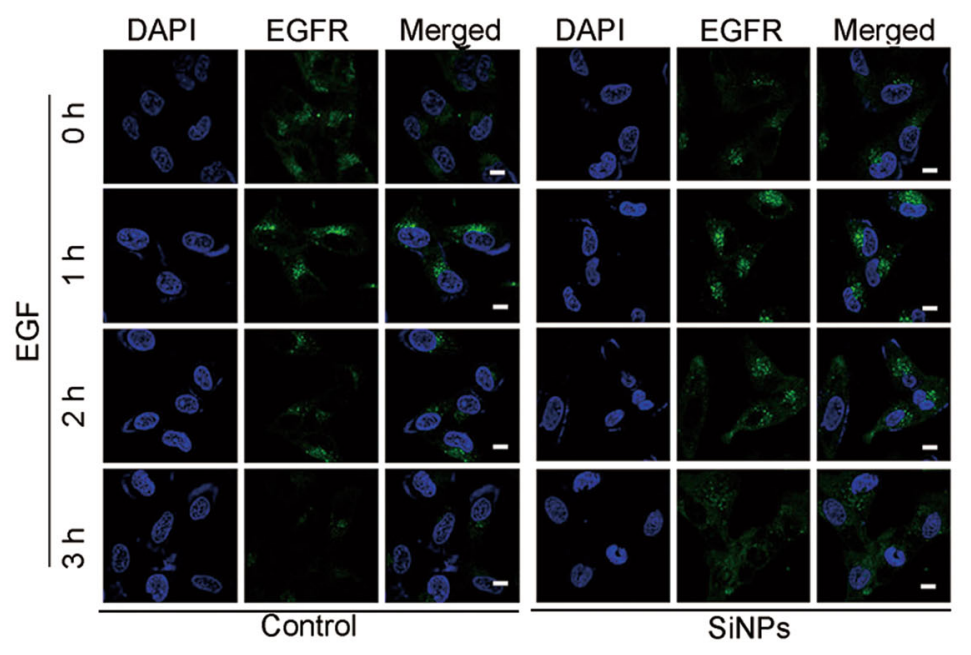

C

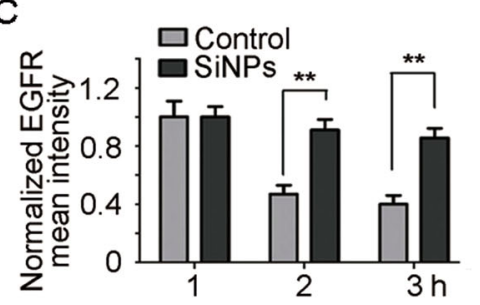

D

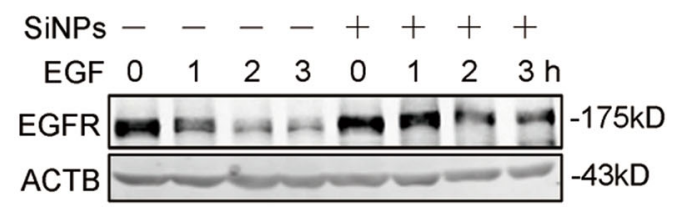

Fig. 5 SiNPs impair lysosomal degradative capacity. a A549 cells transiently expressing CFP-LC3 and YFP-LAMP1 were either starved or treated with SiNPs at $50 \mu \mathrm{g} / \mathrm{mL}$ for $12 \mathrm{~h}$ or $24 \mathrm{~h}$. The cells were then fixed and imaged by confocal microscopy. The graph on the right shows the statistics of the colocalization coefficients of LC3-CFP and LAMP1-YFP. Scale bars: $5 \mu \mathrm{m}$. b A549 cells treated with $50 \mu \mathrm{g} / \mathrm{mL}$ of SiNPs for $24 \mathrm{~h}$ were pre-incubated with $100 \mathrm{ng} / \mathrm{mL}$ of EGF for $15 \mathrm{~min}$, and then the EGF was removed. At the indicated time after EGF treatment, the cells were fixed and

immunostained with anti-EGFR antibody. Scale bars: $10 \mu \mathrm{m}$. c Quantitative analysis of panel B was performed by normalization to the intensity of cells with EGF treatment for $1 \mathrm{~h}(n=30)$. d The EGFR protein level from the cells treated as in $(\mathbf{b})$ was detected by western blot. Data are presented as mean $\pm \mathrm{SEM},{ }^{* *} P<0.01$

of SiNPs-induced PF. To verify our in vitro findings, we examined the key molecule and core process in SiNPstreated mice. Both immunoblotting and IHC assays showed increased p62 expression levels in the lung tissues (Fig. 8a, b), confirming that SiNPs inhibit autophagic degradation in vivo. We next interrogated whether enhancing autophagic flux in vivo could attenuate SiNPs-induced PF. For this purpose, intratracheally instilled mice were treated with rapamycin to activate autophagy and enhance autophagic flux ${ }^{32}$. Rapamycin improved pulmonary compliance while reduced HYP content, collagen deposition, as well as $\alpha-$ SMA levels in mice treated with SiNPs (Fig. 8c, d, e). These data demonstrate that enhancement of autophagic degradation could attenuate the SiNPs-induced $\mathrm{PF}$, and further testify inhibition of autophagic degradation is involved in the pathogenesis of SiNPsinduced PF. 

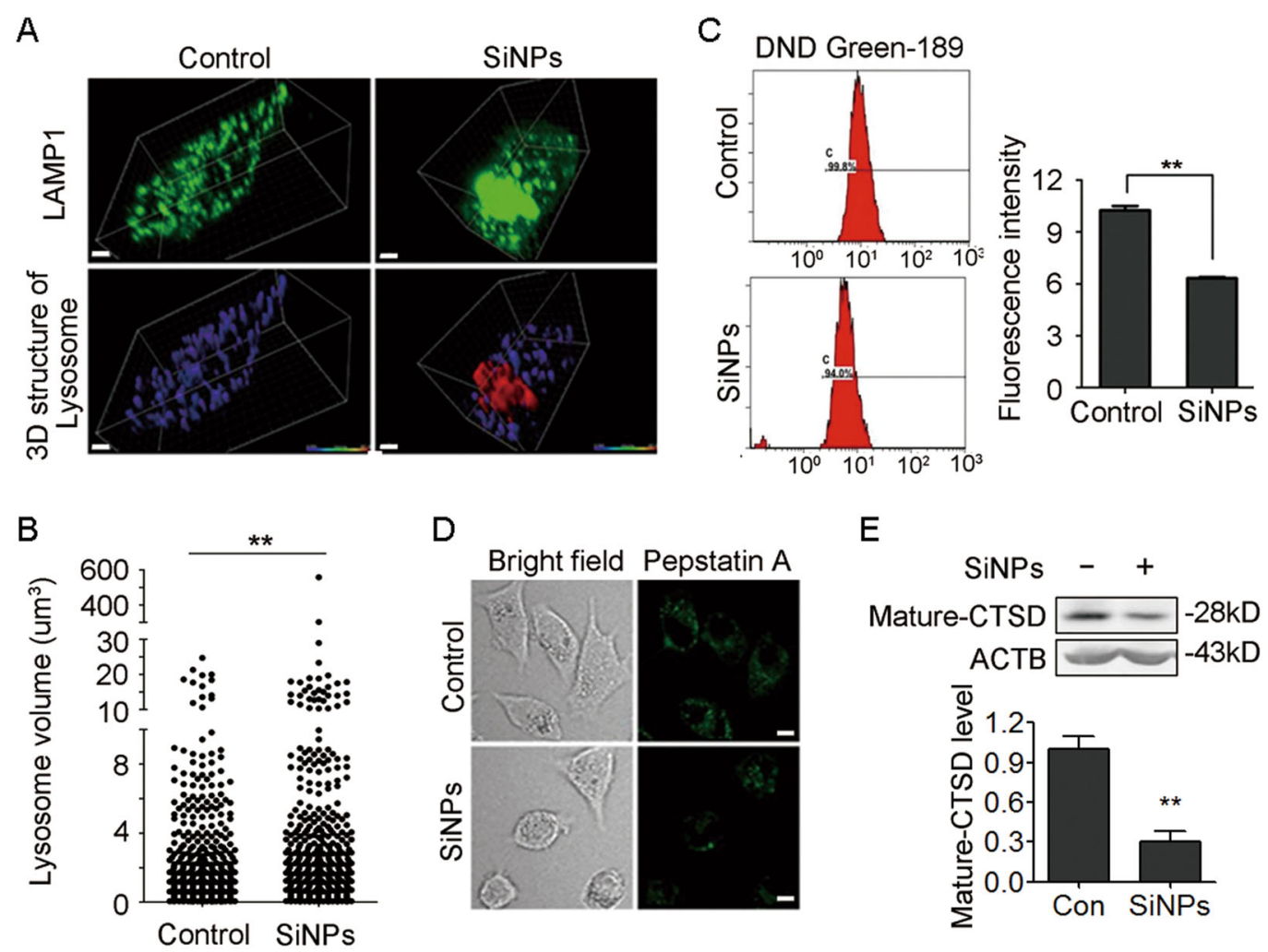

E

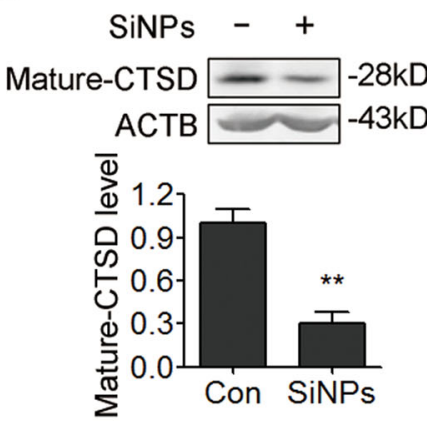

Fig. 6 SiNPs enlarge lysosomes and inhibit lysosomal acidification. a A549 cells transiently expressing YFP-LAMP1 were treated with SiNPs at $50 \mathrm{\mu g} / \mathrm{mL}$ for $24 \mathrm{~h}$. The cells were then fixed and imaged by confocal microscopy. The 3D structure of the lysosome were restructured with Imaris software. Scale bars: $4 \mu \mathrm{m}$. b The lysosome volumes of the cells treated as in (a) were quantified, and the difference between control and SiNPs treatment groups was tested with a Wilcoxon rank sum test. c A549 cells were treated with SiNPs at $50 \mu \mathrm{g} / \mathrm{mL}$ for $24 \mathrm{~h}$, then stained with Lysosensor Green DND-189. The lysosensor fluorescence intensity was detected by flow cytometry. A representative flow cytometry profile is shown on the left. Quantification shown on the right represents the fluorescence intensity of the lysosensor probe. d A549 cells treated with SiNPs at $50 \mu \mathrm{g} / \mathrm{mL}$ for $24 \mathrm{~h}$ were incubated with $1.0 \mathrm{mM}$ Bodipy-FL-pepstatin A for $1 \mathrm{~h}$, and then visualized by confocal microscopy. Scale bars: $5 \mu \mathrm{m}$. e The mature CTSD protein level was assessed by western blot after SiNPs treatment as in (d). The bottom panel shows the statistics for relative mature-CTSD protein level. Data are presented as mean \pm SEM, ${ }^{* *} P<0.01$

\section{Discussion}

Although NPs have been reported to trigger PF development, its cellular and molecular mechanisms remain largely unclear. Previous studies mainly focus on the role of inflammation, ROS or cytokines in this process, but ignore the contribution of AEC autophagy and damage. In this study, we systematically investigated the mechanism of autophagy dysfunction in AECs and its role in SiNPsinduced PF. The major findings of our study include: (a) SiNPs entered AECs and resulted in autophagy dysfunction and apoptosis; (b) Autophagosome accumulation resulted from not only autophagy induction but also autophagic flux inhibition. Furthermore, SiNPs suppressed autophagic degradation by inhibiting lysosomal acidification and thus triggered apoptosis in AECs; (c) the reacidification of lysosome by cAMP cocktail or rapamycin enhanced autophagic flux, and protected AECs from apoptosis under SiNPs treatment; and (d) in vivo enhancement of autophagic flux by rapamycin attenuated
SiNPs-induced apoptosis and PF. These findings demonstrate that impared autophagic degradation of AECs by SiNPs is involved in the progression of PF. Overall, our study elucidates a new mechanism in SiNPs-induced PF, namely that SiNPs inhibit autophagic degradation of AECs by impairing lysosomal acidification, thereby lead to apoptosis of AECs, and initiate PF, which can be rescued by enhancement of autophagic degradation (Fig. 9).

It is generally believed that NPs can target various cell types in lung tissue due to its nano-scale diameter, suggesting there are multiple target cells and mechanisms of action in NPs-induced PF. As a matter of fact, AEC injury and pneumocyte apoptosis have been reported to play roles in the development of $\mathrm{PF}$, and the extent of AEC injury and lack of sufficient repair are critical determinants of PF following exposure to a wide variety of noxious agents ${ }^{33-35}$. However, the involvement of AEC apoptosis in NPs-induced PF has not been investigated. In this study, we not only provided evidence to support the 


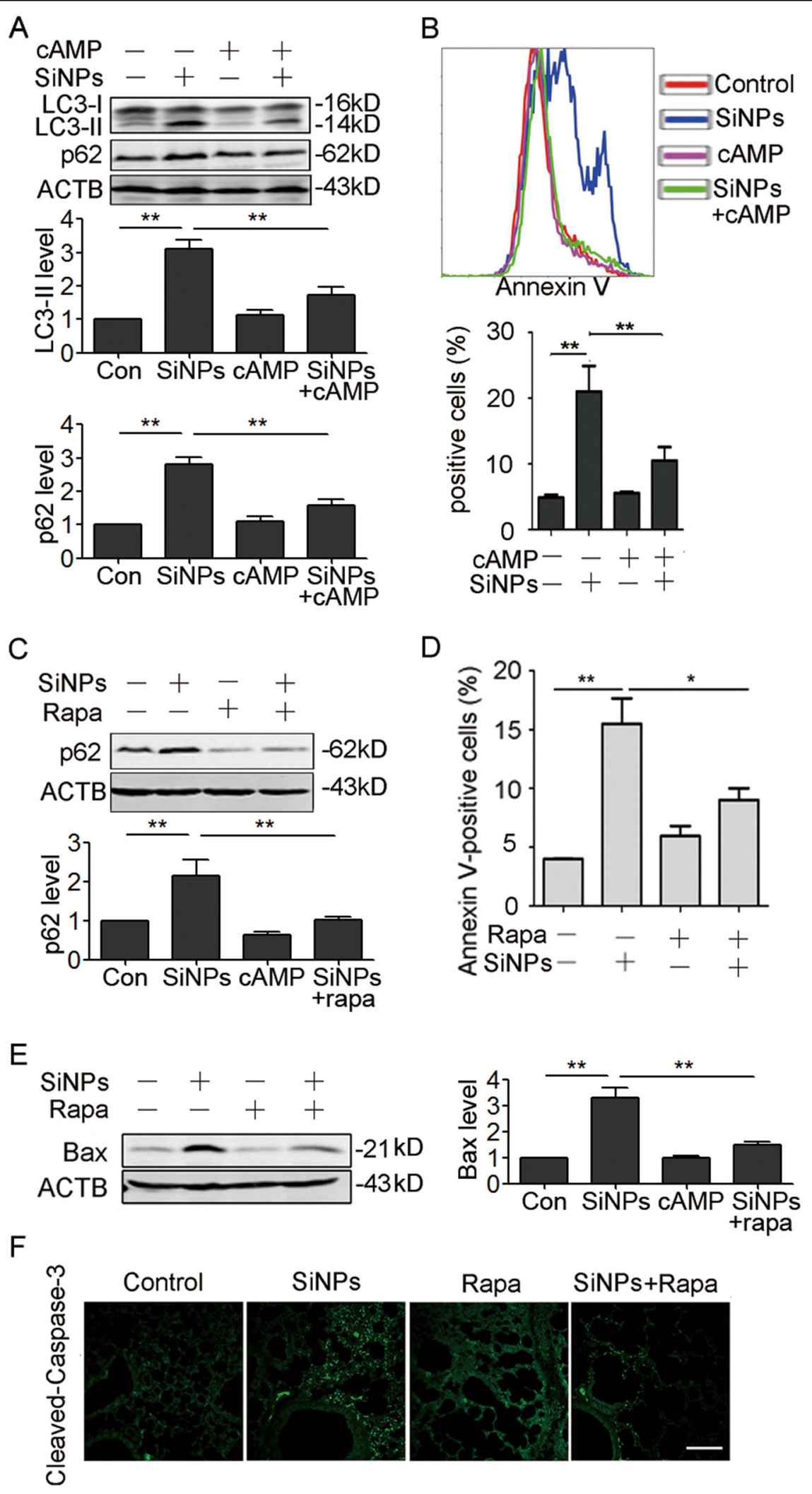

Fig. 7 (See legend on next page.) 
(see figure on previous page)

Fig. 7 Increase of autophagic degradation protects cells from apoptosis. a A549 cells were exposed to SiNPs at $50 \mu \mathrm{g} / \mathrm{mL}$, cAMP cocktail, or both. The expression of corresponding proteins was detected by western blot. The bottom panels show the statistics for relative LC3-II and p62 protein level. b A549 cells were treated with $50 \mu \mathrm{g} / \mathrm{mL}$ of SiNPs for $24 \mathrm{~h}$ in the presence or absence of cAMP cocktail. Cell apoptosis was monitored by flow cytometry analysis with Annexin V-fluorescein isothiocyanate (FITC) (top). The Annexin V positive cells were quantified (bottom). c, d A549 cells were treated with SiNPs in the presence or absence of rapamycin. $\mathbf{c}$ The p62 protein level was detected by western blot, and the bottom panel shows the statistical analysis for relative p62 protein level. $\mathbf{d}$ Cell apoptosis was monitored by Annexin V-FITC with flow cytometry analysis, and the Annexin $V$ positive cells were quantified. $\mathbf{e}, \mathbf{f}$ The mice instilled with SiNPs in the presence or absence of rapamycin for one month were harvested. e Bax expression in lung tissue was detected by western blot. The right panel shows the statistics for relative Bax protein level. $\mathbf{f}$ The Cleaved-Caspase3 expression in lung tissue were detected by IF. Data are presented as mean \pm SEM, ${ }^{*} P<0.05,{ }^{* *} P<0.01$. Scale bars: $100 \mu \mathrm{m}$

alveolar type II cells as a target cell of SiNPs, but also elucidate a unique mechanism of SiNPs-induced cell apoptosis and subsequent PF. Based on our finding, we may conclude that SiNPs lead to AECs apoptosis through autophagy dysfunction, thus adding new knowledge to SiNPs-induced PF.

Autophagy have been implicated in a wide range of pulmonary disease, including $\mathrm{PF}^{36}$. In in vitro models, Araya et al. found that autophagy inhibition resulted in AEC senescence and myfibroblast differentiation, suggesting that insufficient autophagy is an underlying mechanism of both accelerated cellular senescence and myofibroblast differentiation in a cell-type-specific manner $^{37}$. Using Autophagy-related $4 b$ (Atg4b)-deficient mouse model, Cabrera et al. provided the first in vivo evidence that impaired autophagy augmented apoptosis predominantly in alveolar and bronchiolar epithelial cells, indicating that the ATG4B protease and autophagy play a crucial role in protecting epithelial cells against bleomycin-induced stress and apoptosis ${ }^{18}$. However, whether and how dysfunction of autophagy contributes to NPs-induced PF is still undefined. In this study, we demonstrated that accumulation of LC3-II protein in SiNPs-treated AECs were resulted from inhibition of autophagic flux and dysfunction of autolysosome degradation, indicating impairment of autophagy accounts for the AEC apoptosis and PF. Furthermore, we not only attested that SiNPs blocked autophagic degradation, but also elucidated that the underlying mechanism is lysosomal acidification inhibition. Wang et al. reported SiNPsinduced autophagy dysfunction via lysosomal impairment in hepatocytes, suggesting that autophagic degradation inhibition might be a common mechanism in various types of cells exposed to SiNPs ${ }^{38}$. Thus, we hypothesize SiNPs also damage other cells types (e.g., macrophages, fibroblasts) via autophagy dysfunction and contributed to SiNPs-induced PF. The other NPs, such as zinc oxide, gold nanoparticles as well as single-walled carbon nanotubes also damaged lysosome, therefore inhibited autophagic flux to lead to cytotoxicity, indicating that autophagy dysfunction resulting from lysososmal impairment acts as a general toxic mechanism of NPs ${ }^{39-41}$. It also should be noted that SiNPs upregulated $L C 3 \mathrm{mRNA}$ level, leading to more LC3 than CQ treatment, suggesting that SiNPs not only impared autophagic degradation, but also played a role in autophagy induction (Figure S4).

Interestingly, our finding is similar to the so-called lysosomal storage disorders (LSDs), which are a heterogeneous group of inherited diseases, resulting from deficiencies of one or more enzymes or transporters that normally reside within the lysosomes ${ }^{42}$. In several LSDs, the accumulation of autophagosomes has been contributed to a decreased autophagic flux ${ }^{43,44}$. Furthermore, perturbations of autophagic flux by specific gene mutations have been suggested to contribute to the disease etiology of specific LSDs. Based on lysosomal dysfunction and decreased autophagic degradation, SiNPs-induced PF might be defined as a type of LSD-like disease ${ }^{45}$. Collectively, the LSD-like diseases are caused by various stimuli that impair the lysosome, inhibit autophagic flux and thus trigger subsequent injury.

NPs exposure could lead to PF, therefore, it is important to develop intervention measures for prevention and treatment of NPs-stimulated PF. Currently, the treatment of PF continues to pose major difficulties. Until recently, most treatment options have focused on inflammation by using anti-inflammatory agents ${ }^{46}$. However, the strategy has been found to be effective only in small groups of patients ${ }^{47}$. More new targets, for example, PF-associated cytokines, have been deciphered along with more understanding of the underlying pathophysiology of PF. However, because of multiple routes for PF stimulation, blocking one of them had no significant effect on the ultimate outcome ${ }^{48}$. Promoting the prevention of epithelial damage/healing of the lung epithelium has been increasingly investigated as a potential approach by which subsequent inflammation, remodeling and fibrosis can be abrogated. Therefore, there is a need to develop therapies that promote the protection and health of lung epithelial cells. In the present study, we paid our attention to AEC damage under SiNPs treatment, and clarified the underlying mechanism of autophagy dysfunction in AEC damage, thus enriched our understanding of the pathogenesis of this disease. Therefore, our results provide not only a new strategy targeting autophagy flux blockage to prevent SiNPs-induced AEC damage and PF, but also experimental data for a preventive effect of 
A

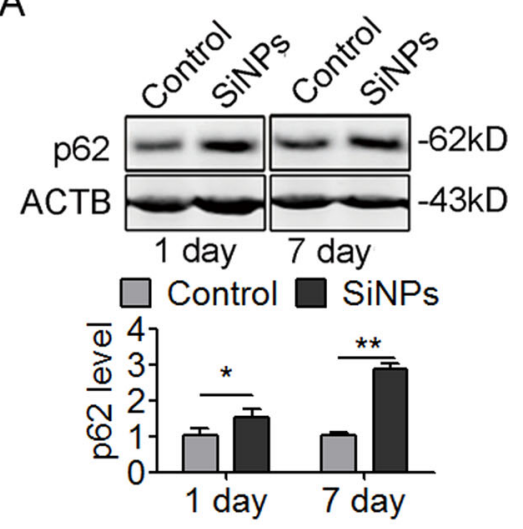

C

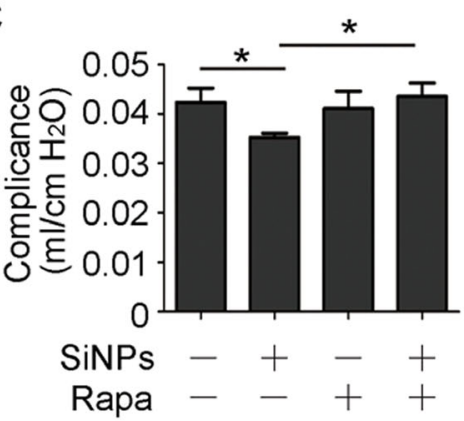

B

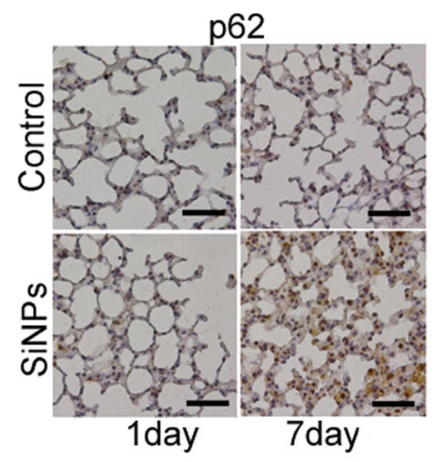

D

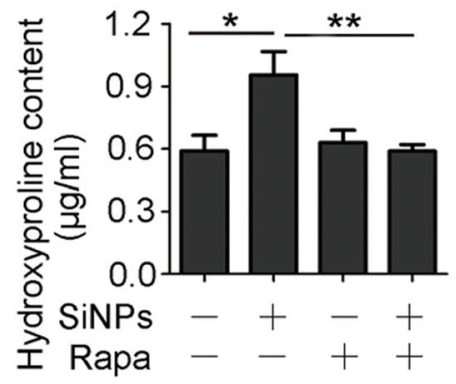

$\mathrm{E}$

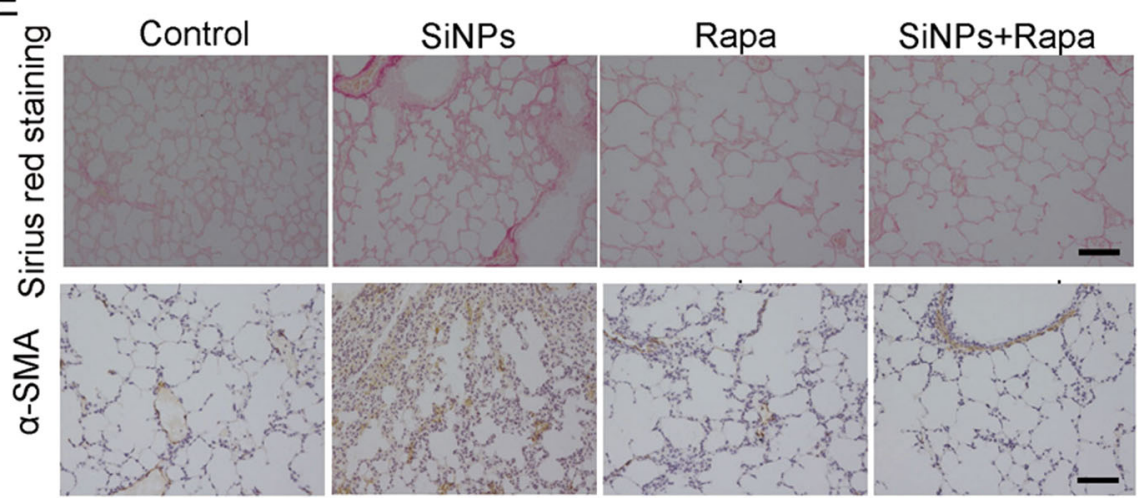

Fig. 8 A decrease in autophagic degradation participates in SiNPs-induced PF. a, b The mice were instilled with PBS or SiNPs and harvested at the indicated time points. a p62 protein expression in lung tissue was detected by western blot. The bottom panel shows the statistics for the relative p62 protein level. $\mathbf{b}$ The expression of p62 was detected by IHC at 1 day or 7 days after instillation. $\mathbf{c}-\mathbf{e}$ Mice were instilled with SiNPs in the presence or absence of rapamycin and harvested 1 month later. $\mathbf{c}$ Lung compliance was determined by Buxco system. $\mathbf{d}$ The HYP content in lung tissue was assessed by spectrophotometry. e The lung section was stained by Sirius red staining or performed IHC to detect a-SMA. Data are presented as mean \pm SEM $\left(n=4\right.$ for each group), ${ }^{*} P<0.05,{ }^{*} P<0.01$. Scale bars: $100 \mu \mathrm{m}$

autophagic degradation enhancement with rapamycin in SiNPs-treated mice.

In summary, for the first time, we have shown that autophagy participates in SiNPs-induced PF. We have also identified that the autophagic flux blockage results from lysosomal acidification inhibition, which then triggers apoptosis in AECs and subsequent PF. These findings provide a new mechanism by which SiNPs trigger PF by targeting AECs. Furthermore, these results may lead to new strategies to prevent SiNPs-induced PF by enhancing autophagic degradation.

\section{Acknowledgements}

We thank Prof. Wei Liu for sharing plasmids, Prof. Yuehai Ke for providing cell lines, and Prof. Zhihua Chen for critical reading of the manuscript. The authors are grateful to Miss Shuangshuang Liu at the core facility for help with imaging. This manuscript was polished by Textcheck. This work was supported by the National Natural Science Foundation of China (31570786, 81703255, 81672847); Nantong Jiangsu scientific research project (MS12017014-8). 


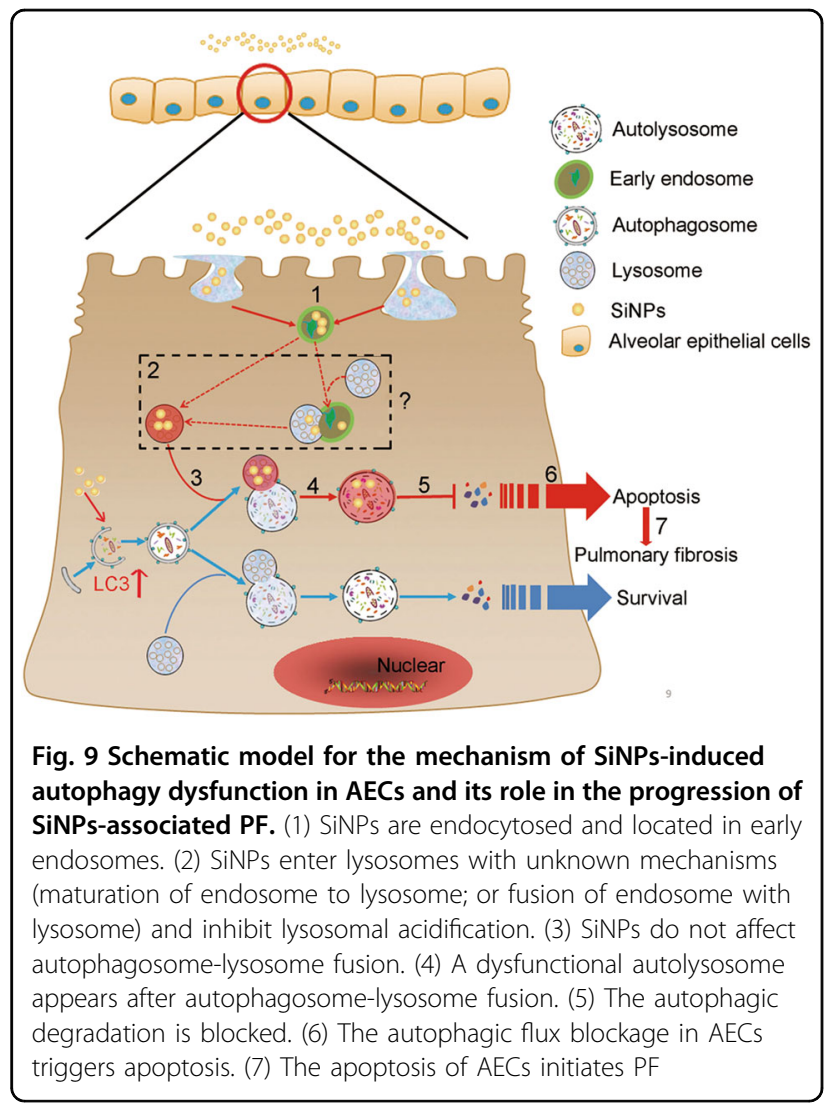

\section{Authors' contributions}

All authors read and approved the manuscript. X.Z. and S.W. did most of the experiments and prepared tables and figures; Z.Z. and Q.J. provided SiNPs and characterized it; X.Z., C.L., S.W., D.S., and R.B. performed animal experiments; X. G., G.C., and Z.X. designed the study; X.Z., X.G., G.C., and Z.X. prepared the manuscript and figures.

\section{Author details}

'Institute of Environmental Medicine, Zhejiang University School of Medicine, Hangzhou 310058, China. ${ }^{2}$ Department of Occupational Medicine and Environmental Toxicology, School of Public Health, Nantong Unversity, Nantong 226019, China. ${ }^{3}$ The First Affiliated Hospital, Zhejiang University School of Medicine, Hangzhou 310058, China. ${ }^{4}$ State Key Laboratory of Modern Optical Instrumentation, Centre for Optical and Electromagnetic Research, JORCEP (Sino-Swedish Joint Research Center of Photonics), Zhejiang University, Hangzhou 310058, China. ${ }^{5}$ Collaborative Innovation Center for Diagnosis and Treatment of Infectious Diseases, Zhejiang University, Hangzhou 310058, China

\section{Conflict of interest}

The authors declare that they have no conflict of interest.

\section{Publisher's note}

Springer Nature remains neutral with regard to jurisdictional claims in published maps and institutional affiliations.

Supplementary Information accompanies this paper at (https://doi.org/ 10.1038/s41419-019-1340-8).
Received: 13 November 2018 Revised: 18 December 2018 Accepted: 7 January 2019

Published online: 12 February 2019

\section{References}

1. Nel, A., Xia, T., Madler, L. \& Li, N. Toxic potential of materials at the nanolevel. Science 311, 622-627 (2006).

2. Bapat, G., Labade, C., Chaudhari, A. \& Zinjarde, S. Silica nanoparticle based techniques for extraction, detection, and degradation of pesticides. Adv. Colloid Interface Sci. 237, 1-14 (2016).

3. Guo, C. et al. Silica nanoparticles promote oxLDL-induced macrophage lipid accumulation and apoptosis via endoplasmic reticulum stress signaling. Sci. Total Environ. 631-632, 570-579 (2018).

4. Mebert, A. M., Baglole, C. J., Desimone, M. F. \& Maysinger, D. Nanoengineered silica: properties, applications and toxicity. Food Chem. Toxicol. 109(Pt 1), 753-770 (2017).

5. Oberdorster, G. Safety assessment for nanotechnology and nanomedicine: concepts of nanotoxicology. J. Intern. Med. 267, 89-105 (2010).

6. Wuyts, W. A. et al. The pathogenesis of pulmonary fibrosis: a moving target. Eur. Respir. J. 41, 1207-1218 (2013).

7. Sun, B. et al. NADPH oxidase-dependent NLRP3 inflammasome activation and its important role in lung fibrosis by multiwalled carbon nanotubes. Small 11, 2087-2097 (2015).

8. Azad, N. et al. Reactive oxygen species-mediated p38 MAPK regulates carbon nanotube-induced fibrogenic and angiogenic responses. Nanotoxicology $\mathbf{7}$, 157-168 (2013).

9. Yang, H., Wu, Q. Y., Li, M. Y., Lao, C. S. \& Zhang, Y. J. Pulmonary toxicity in rats caused by exposure to intratracheal instillation of $\mathrm{SiO}_{2}$ nanoparticles. Biomed. Environ. Sci. 30, 264-279 (2017).

10. Chang, X. H. et al. Nickel oxide nanoparticles induced pulmonary fibrosis via TGF- beta1 activation in rats. Hum. Exp. Toxicol. 36, 802-812 (2017).

11. Saputra, D. et al. Inhalation of carbon black nanoparticles aggravates pulmonary inflammation in mice. Toxicol. Res. 30, 83-90 (2014).

12. Chapman, H. A. Epithelial-mesenchymal interactions in pulmonary fibrosis. Annu. Rev. Physiol. 73, 413-435 (2011).

13. Furuyama, A., Kanno, S., Kobayashi, T. \& Hirano, S. Extrapulmonary translocation of intratracheally instilled fine and ultrafine particles via direct and alveolar macrophage-associated routes. Arch. Toxicol. 83, 429-437 (2009).

14. Wottrich, R., Diabate, S. \& Krug, H. F. Biological effects of ultrafine model particles in human macrophages and epithelial cells in mono- and co-culture. Int. J. Hyg. Environ. Health 207, 353-361 (2004).

15. Romeo, Y. \& Lemaitre, B. Autophagy in immunity and inflammation. Nature 469, 323-335 (2011)

16. Gui, Y. S. et al. mTOR overactivation and compromised autophagy in the pathogenesis of pulmonary fibrosis. PLOS. ONE. 10, e0138625 (2015).

17. Singh, K. K. et al. The essential autophagy gene ATG7 modulates organ fibrosis via regulation of endothelial-to-mesenchymal transition. J. Biol. Chem. 290, 2547-2559 (2015).

18. Cabrera, S. et al. Essential role for the ATG4B protease and autophagy in bleomycin-induced pulmonary fibrosis. Autophagy 11, 670-684 (2015).

19. Yang, H. Z. et al. TLR4 activity is required in the resolution of pulmonary inflammation and fibrosis after acute and chronic lung injury. Am. J. Pathol. 180, 275-292 (2012).

20. Guo, Y. J. et al. AP1 is essential for generation of autophagosomes from the trans-Golgi network. J. Cell. Sci. 125, 1706-1715 (2012).

21. Huang, R. et al. Deacetylation of nuclear LC3 drives autophagy initiation under starvation. Mol. Cell 57, 456-466 (2015).

22. Qian, J. et al. Photosensitizer encapsulated organically modified silica nanoparticles for direct two-photon photodynamic therapy and In Vivo functional imaging. Biomaterials 33, 4851-4860 (2012).

23. Qian, J. et al. Bio-molecule-conjugated fluorescent organically modified silica nanoparticles as optical probes for cancer cell imaging. Opt. Express 16, 19568-19578 (2008)

24. Choi, M. et al. Transient pulmonary fibrogenic effect induced by intratracheal instillation of ultrafine amorphous silica in AJJ mice. Toxicol. Lett. 182, 97-101 (2008).

25. Gao, X. W. et al. Nucleolar follistatin promotes cancer cell survival under glucose-deprived conditions through inhibiting cellular rRNA synthesis. J. Biol. Chem. 285, 36857-36864 (2010). 
26. Fu, Q., Tao, Y., Piao, H. L., Du, M. R. \& Li, D. J. Trophoblasts and decidual stromal cells regulate decidual NK cell functions via interaction between collagen and LAIR-1. Am. J. Reprod. Immunol. 71, 368-378 (2014).

27. Gao, X. W. et al. Reduction of AUF1-mediated follistatin mRNA decay during glucose starvation protects cells from apoptosis. Nucleic Acids Res. 42, 10720-U10806 (2014).

28. Ezzie, M. E. et al. Thrombospondin-1-deficient mice are not protected from bleomycin-induced pulmonary fibrosis. Am. J. Respir. Cell Mol. Biol. 44, 556-561 (2011).

29. Yao, R. et al. Adiponectin attenuates lung fibroblasts activation and pulmonary fibrosis induced by paraquat. PLOS. ONE. 10, e0125169 (2015).

30. Chen, C. S., Chen, W. N., Zhou, M., Arttamangkul, S. \& Haugland, R. P. Probing the cathepsin D using a BODIPY FL-pepstatin A: applications in fluorescence polarization and microscopy. J. Biochem. Biophys. Methods 42, 137-151 (2000).

31. Coffey, E. E., Beckel, J. M., Laties, A. M. \& Mitchell, C. H. Lysosomal alkalization and dysfunction in human fibroblasts with the Alzheimer's disease-linked presenilin 1 A246E mutation can be reversed with CAMP. Neuroscience $\mathbf{2 6 3}$ 111-124 (2014).

32. Abdulrahman, B. A. et al. Autophagy stimulation by rapamycin suppresses lung inflammation and infection by Burkholderia cenocepacia in a model of cystic fibrosis. Autophagy 7, 1359-1370 (2011).

33. Liu, G., Beri, R., Mueller, A. \& Kamp, D. W. Molecular mechanisms of asbestosinduced lung epithelial cell apoptosis. Chem.-Biol. Interact. 188, 309-318 (2010).

34. Thomas, A. Q. et al. Heterozygosity for a surfactant protein C gene mutation associated with usual interstitial pneumonitis and cellular nonspecific interstitial pneumonitis in one kindred. Am. J. Resp. Crit. Care 165, 1322-1328 (2002).

35. Hagimoto, N., Kuwano, K., Nomoto, Y., Kunitake, R. \& Hara, N. Apoptosis and expression of Fas/Fas ligand mRNA in bleomycin-induced pulmonary fibrosis in mice. Am. J. Resp. Cell Mol. 16, 91-101 (1997).
36. $\mathrm{Hu}, \mathrm{Y}$. et al. mTOR and autophagy in regulation of acute lung injury: a review and perspective. Microbes Infect. 16, 727-734 (2014).

37. Araya, J. et al. Insufficient autophagy in idiopathic pulmonary fibrosis. Am. J. Physiol.-Lung C. 304, L56-L69 (2013).

38. Wang, J. et al. Silica nanoparticles induce autophagy dysfunction via lysosomal impairment and inhibition of autophagosome degradation in hepatocytes. Int. J. Nanomed. 12, 809-825 (2017).

39. Wang, B. et al. The size of zinc oxide nanoparticles controls its toxicity through impairing autophagic flux in A549 lung epithelial cells. Toxicol. Lett. 285, 51-59 (2018).

40. Ma, X. et al. Gold nanoparticles induce autophagosome accumulation through size-dependent nanoparticle uptake and lysosome impairment. ACS Nano 5, 8629-8639 (2011).

41. Wan, B. et al. Single-walled carbon nanotubes and graphene oxides induce autophagosome accumulation and lysosome impairment in primarily cultured murine peritoneal macrophages. Toxicol. Lett. 221, 118-127 (2013).

42. Xu, H. X. \& Ren, D. J. Lysosomal physiology. Annu. Rev. Physiol. 77, 57-80 (2015)

43. Lim, J. A., Kakhlon, O., Li, L., Myerowitz, R. \& Raben, N. Pompe disease: shared and unshared features of lysosomal storage disorders. Rare Dis. 3, e1068978 (2015).

44. Motta, M. et al. Clinical, biochemical and molecular characterization of prosaposin deficiency. Clin. Genet. 11, 2075-2086 (2016).

45. Ward, C. et al. Autophagy, lipophagy and lysosomal lipid storage disorders. Bba-Mol. Cell Biol. L 1861, 269-284 (2016).

46. Behr, J. \& Richeldi, L. Recommendations on treatment for IPF. Respir. Res. 14 (Suppl 1), S6 (2013).

47. Sterclova, M. \& Vasakova, M. Promising new treatment targets in patients with fibrosing lung disorders. World J. Clin. Cases 2, 668-675 (2014).

48. du Bois, R. M. Strategies for treating idiopathic pulmonary fibrosis. Nat. Rev Drug. Discov. 9, 129-140 (2010). 